\title{
A Sequential Block Based SLM Approach for Papr and Interference Reduction of FBMC/OQAM Systems
}

\author{
${ }^{1}$ B. Kedarnath, ${ }^{2}$ Dr. Shah Aqeel. A, ${ }^{3}$ Dr. Syed Abdul Sattar \\ ${ }^{1}$ Research scholar, Department of ECE, Rayalaseema University, Kurnool, A.P, India \\ ${ }^{2}$ Principal, M.M.A.N.C., Mansoora Malegaon, Nasik, M.S. India \\ ${ }^{3}$ Director R \& D, Professor ECE, Nawab Shah Alam Khan College of Engineering and Technology, \\ Hyderabad, T.S, India
}

\begin{abstract}
Filter bank multi carrier (FBMC) is a modulation technique with enhanced spectral properties and also finds an alternate solution for cyclic prefix based OFDM system in coordination with offset quadrature amplitude modulation (OQAM). Peak to average power ratio (PAPR) is still a defined problem in this system; in this paper a modified selective mapping (SLM) approach is proposed for the reduction of PAPR and selection of optimal candidate signal. The proposed approach experimental results reveal that this approach is performing better than tradition FBMC-SLM approaches under different constraints.
\end{abstract}

Keywords: $O F D M, F B M C, O Q A M, P A P R, O B I$

\section{INTRODUCTION}

Since three decades OFDM is widely adopted in many of the broadband wired and wireless communication systems because of its high range of advantages over other technologies like carrier and symbol synchronization, low ICI, maximum collection of narrow bands over a limited available bandwidth and many. However, the time frequency resources cannot be assigned flexibly leading to poor performance and spectral behavior for high data rates [1]. Filter bank multi carrier (FBMC) with OQAM provides a solution for poor spectral efficiency by employing the pulses that are localized in both time and frequency. Many properties of FBMC is similar to OFDM and one of the critical issue is implementing of this is its high PAPR value [2].

FBMC with OQAM provides several advantages over traditional OFDM systems like (i) the cyclic prefix is no longer required (ii) the side lobe of its power spectrum density is very low [3]. However this system also suffers from few disadvantages like heavier computation cost due to extra filtering operation, complex channel equalization and channel estimation due to the presence of imaginary interference components. This work is concentrated on reducing PAPR value and to provide good spectral efficiency by reducing the side lobes.

Several works were proposed in past few years on reduction of PAPR for FBMC systems; in [4] Rahim et. al introduced clipping based reduction scheme for OFDM/OQAM systems. However, the bit error rate is increased and also the side lobe. In [5] Kollar et.al, proposed a iterative noise cancellation technique by employing Bus-gang noise cancellation at the receiver but it increased the decoder complexity at the receiver. In [6] Javaudin et.al proposed SLM (selective mapping) approach for reducing PAPR in which the OFDM/OQAM symbol is divided into two parts (real \& imaginary) and adopted phase rotation vectors for the first $2 \mathrm{~K}$ parts (K:length of the prototype filter). Due to the selection of random phase rotation vectors the performance was poor when $K$ is too long. In [7] Yuen et.al, inserted a precoded matrix in between the multiplexer and OFDM/OQAM modulator to reduce PAPR. In [26] and [27], a sliding window tone reservation (SW-TR) technique has been proposed. The SW-TR method utilizes the peak reduction tones of several consecutive data blocks to cancel the peak power of the FBMC/OQAM signals inside a window.

In this paper a progressive SLM approach is proposed to reduce the PAPR while attaining good BER performance and side lobe efficiency. This paper is organized as follows, the first part presents 


\section{B. Kedarnath et al.}

introduction to OFDM/OQAM systems, its importance and the research done so far by different authors. In the second part the topology of OFDM/OQAM system, its architecture and mathematical analysis is presented. Third part presents the proposed approach for minimizing the PAPR. Fourth part presents the experimental results obtained with the proposed approach under different constraints and conditions ending with conclusions.

\section{RELATED WORK}

\subsection{OFDM/OQAM system}

This has drawn much interest in research due to its high spectrum efficiency where the signal is obtained by summing $\mathrm{M}$ time shifted OFDM/OQAM symbols each of which is obtained by passing QAM symbols through a prototype filter [2].

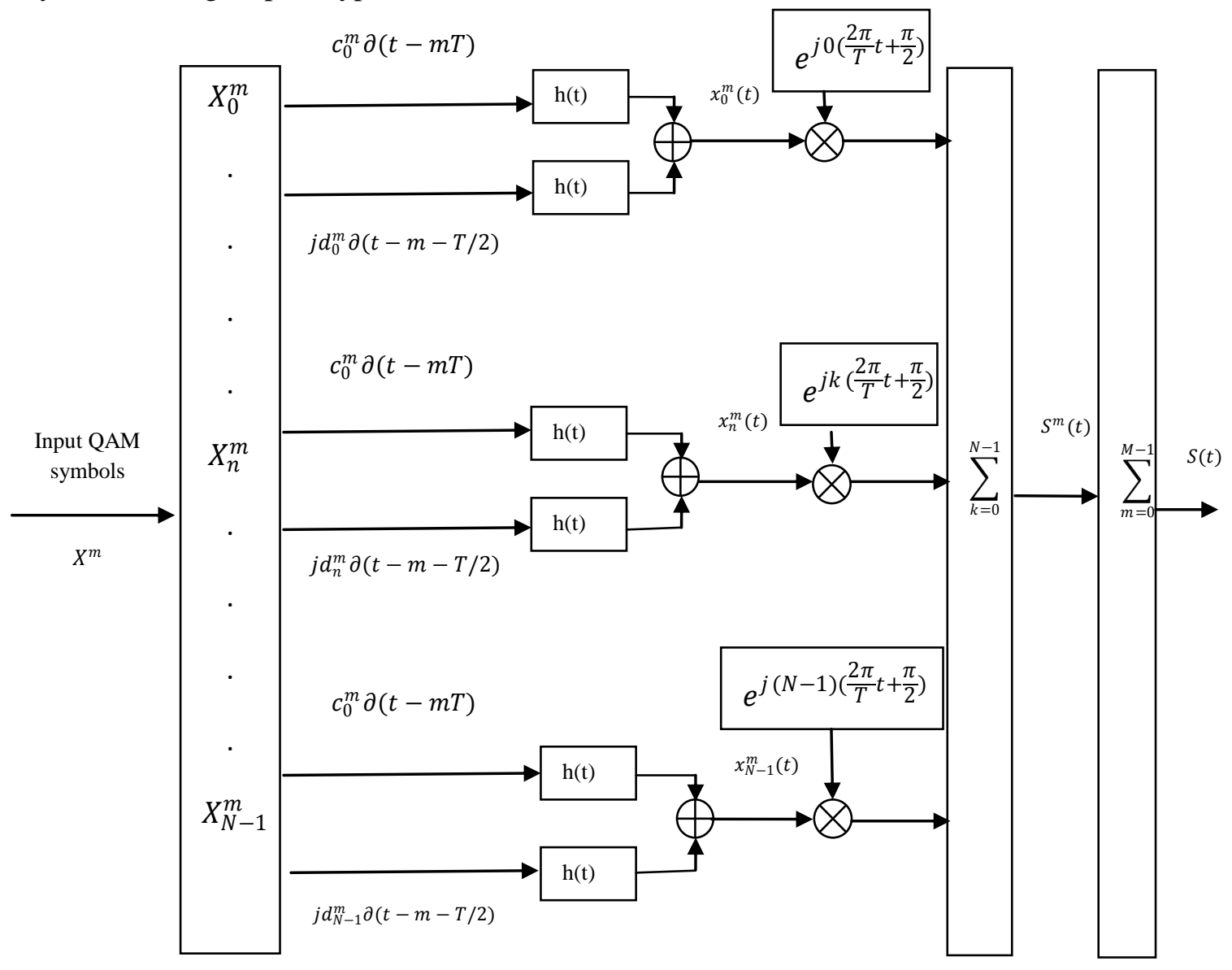

Figure1. Block diagram of OFDM/OQAM system

The OFDM/OQAM transmitter structure is depicted in figure 1 which consists of ' $N$ ' number of sub carriers, after the QAM modulation the input symbols are first converted into parallel form by serial to parallel converter which can be represented as

$X=\left[X^{0}, X^{1}, \ldots \ldots \ldots \ldots, X^{M-1}\right]$

Where ' $\mathrm{M}$ ' is the number of data blocks and $\mathrm{X}^{\mathrm{m}}$ is the $\mathrm{m}^{\text {th }}$ data block which is defined as

$X^{m}=\left[X_{0}^{m}, X_{1}^{m} \ldots \ldots \ldots . X_{N-1}^{m}\right]^{T}$, Where ' $\mathrm{N}$ ' represents the number of subcarriers then

$X_{n}^{m}=c_{n}^{m}+j d_{n}^{m}$

Where 'c' and' $\mathrm{d}$ ' represents the real and imaginary parts of $X_{n}^{m}$. The real and imaginary parts of $X_{n}^{m}$ are staggered by $T / 2$ and passed through the prototype filter to obtain

$x_{n}^{m}(t)=c_{n}^{m} h(t-m T)+j d_{n}^{m}\left(t-\frac{T}{2}-m T\right)$

Where $\mathrm{h}(\mathrm{t})$ is the response of the prototype filter. Here in this paper the prototype filter coefficients is represented below 
$h(t)=\alpha\left(c(0)+2 \sum_{i=1}^{K-1}(-1)^{i} c(i) \cos \left(\frac{2 i \pi}{K N} t\right)\right)$

Where $\alpha$ is the normalization factor and $\mathrm{c}(\mathrm{i}), \mathrm{i}=0,1 \ldots \ldots \mathrm{K}-1$ are given as $\mathrm{c}(0)=1, \mathrm{c}(1)=0.97195$ $\mathrm{c}(2)=0.7071 \mathrm{c}(3)=0.23514$

The modulated symbols in $\mathrm{N}$ orthogonal sub carriers is represented as

$S_{n}^{m}(t)=\left\{c_{n}^{m} h(t-m T)+j d_{n}^{m}\left(t-\frac{T}{2}-m T\right)\right\} e^{j n\left(\frac{2 \pi}{T} t+\frac{\pi}{1}\right)}$

For $\mathrm{K}=0,1,2 \ldots \mathrm{N}-1$. Then $S_{n}^{m}(t)$ on all the $\mathrm{N}$ sub carriers are added up together to obtain

$S^{m}(t)=\sum_{n=0}^{N-1} s_{n}^{m}(t)$

The desired OFDM/OQAM signal is represented as

$s(t)=\sum_{m=0}^{M-1} S^{m}(t)$

For $0 \leq t \leq\left(M+N-\frac{1}{2}\right) T$. Finally the PAPR for the system is represented as

$P A P R=10 \log 10\left(\frac{\max _{p T \leq t \leq\left(p+\frac{1}{2}\right) T}|s(t)|^{2}}{\text { Pave }}\right)$

Where Pave: is the average power of $\mathrm{S}(\mathrm{t})$

\section{Proposed Approach}

The main intension of the proposed approach is to optimize the phase sequences in a sequential manner. By considering previous block symbols may reduce the peak power of the current block that is $S^{0}(t), S^{1}(t), \ldots \ldots . S^{m-1}(t)$ may reduce the peak power of $S^{m}(t)$. Initially at the zeroth block $S^{0}(t)$ by different phase sequences and chose the one with minimum peak power which is then forwarded to the first block which is represented as below

$\min _{b^{1}, u} \max _{2 T \leq t \leq 4 T}\left|\hat{S}^{o}(t)+\sum_{n=0}^{N-1} S_{n}^{1}(t) b_{n}^{1, u}\right|^{2}$

The optimal phase rotation vector is denoted as $\mathrm{b}^{1, \mathrm{u}^{*}}$ and the new symbols generated are represented as

$\hat{S}^{1}(t)=\sum_{n=}^{N-1} S_{n}^{1}(t) b_{n}^{1, u^{*}}$

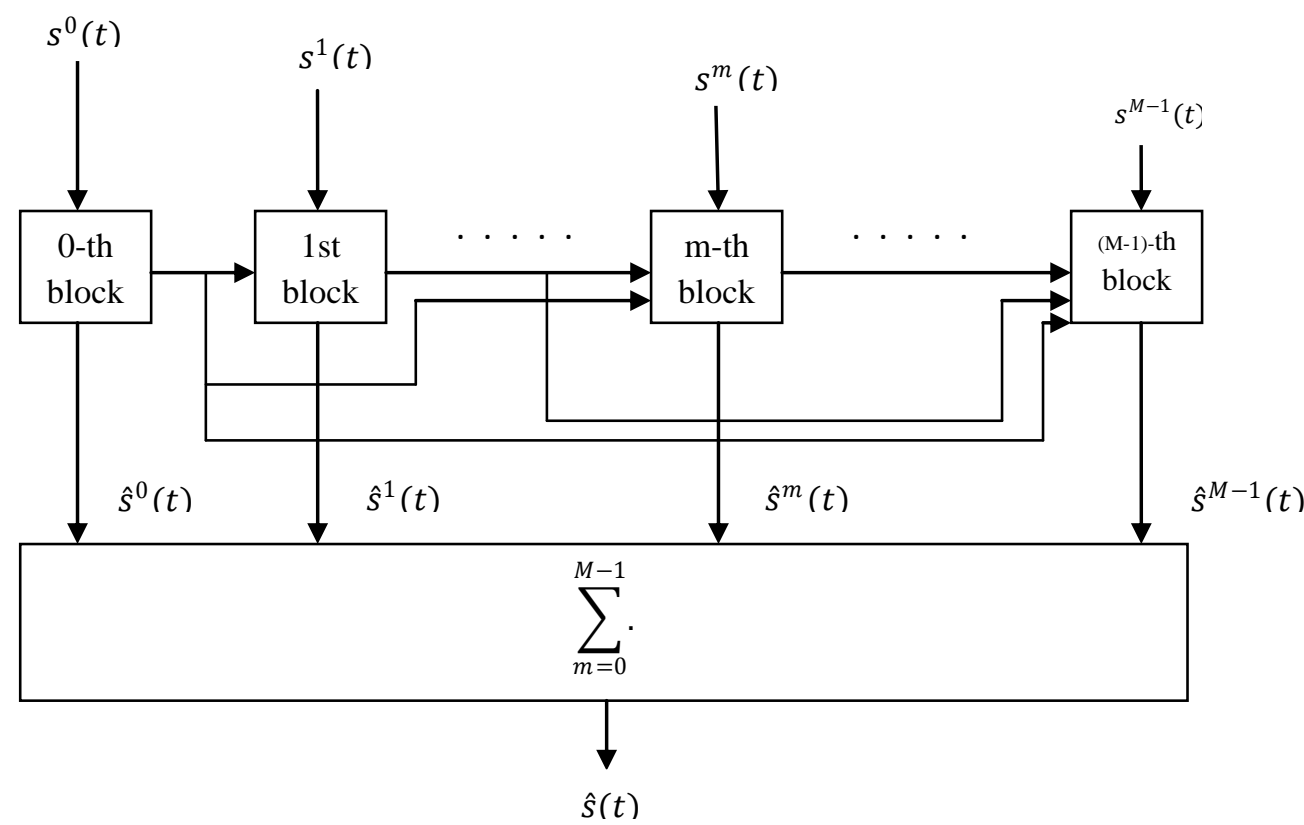

Figure2. Block diagram for the proposed approach

Both these symbols are sent to the second block for the calculation of new symbols and this process is repeated till M-1 blocks, all merge together to for the FBMC/OQAM signal. 


\section{EXPERIMENTAL RESUlts}

Simulations were carried with $\mathrm{N}=256$ sub carriers under 4, 16-QAM modulation schemes. The proposed approach is compared against tradition SLM OFDM [10], PTS OFDM [11] and FBMC SLM [6] approaches. Parameters like CCDF (complementary cumulative distribution function), BER (bit Error Rate) and PSD (power spectral density were considered to evaluate the performance of the proposed approach. The BER performance is evaluated under AWGN (additive white Gaussian noise) environment and Rayleigh fading channel environment.

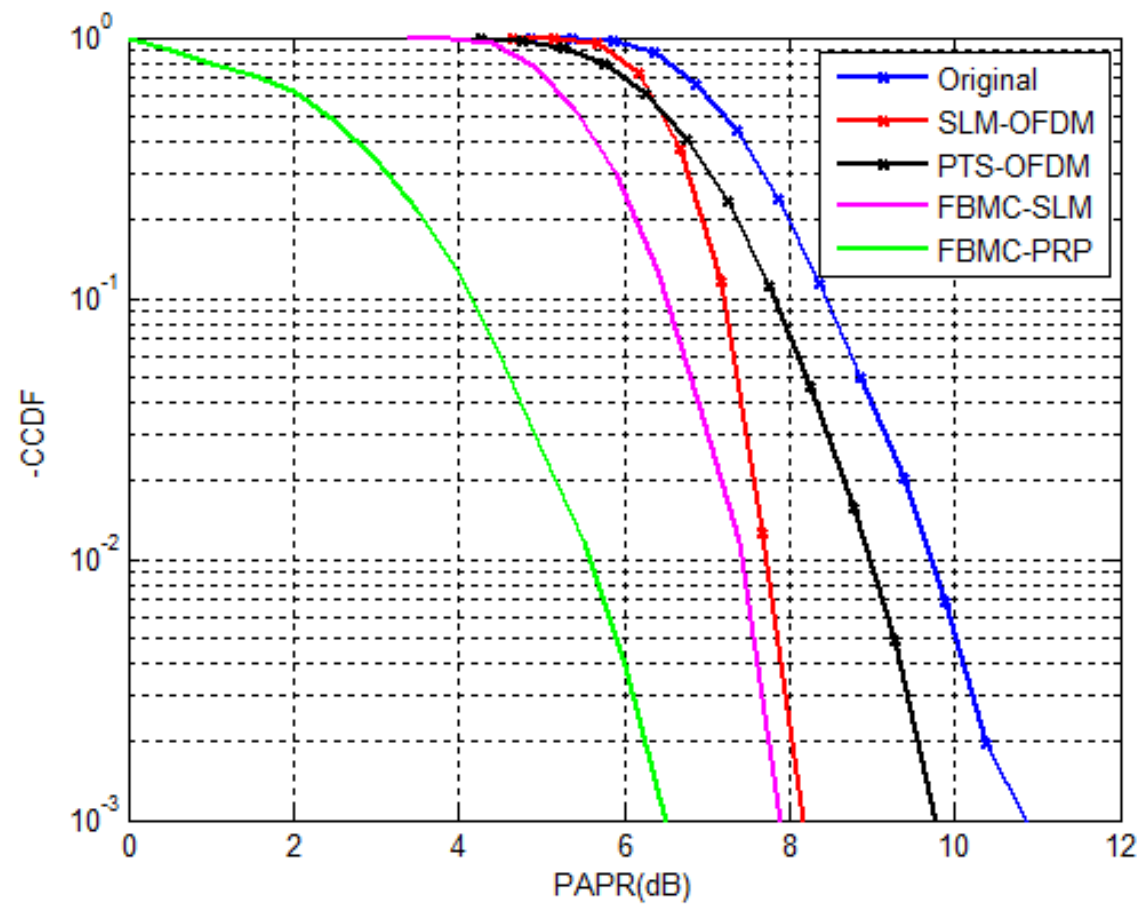

Figure3. $C C D F$ Vs PAPR with $N=256$ and 16-QAM modulation

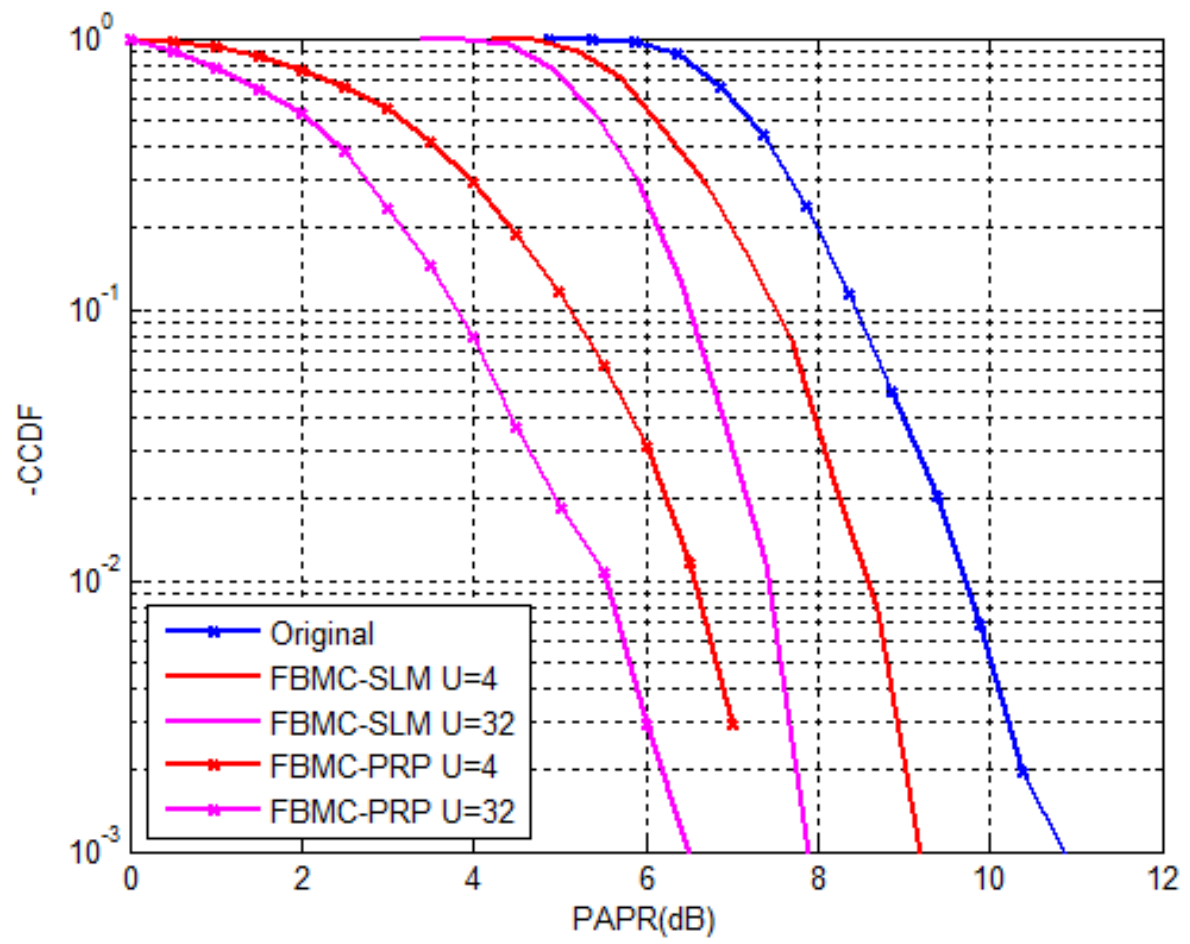

Figure4. $C C D F$ VS $P A P R$ with $N=256$ and at $U=4 \& 32$ for $16-Q A M$

From figure 3 graph it can be observed that the proposed approach is obtaining $0.8 \mathrm{~dB}$ less PAPR than conventional FBMC based SLM approach at CCDF of $10^{-3}$ 
The above figure depicts the PAPR analysis of the proposed approach at different values of ' $U$ ' it is clearly observed that at $\mathrm{U}=32$ it obtaining a CCDF of $10^{-3}$ at $6.2 \mathrm{~dB}$ which is less than $0.7 \mathrm{db}$ when compared with other methods.

From all the experimental results it is evident that the proposed approach of sequential adding of blocks yielding better results in terms of PAPR and the spectrum is utilized efficiently. It can also be observed that it can provide a high spectral efficiency as shown in figure $5 \& 6$.

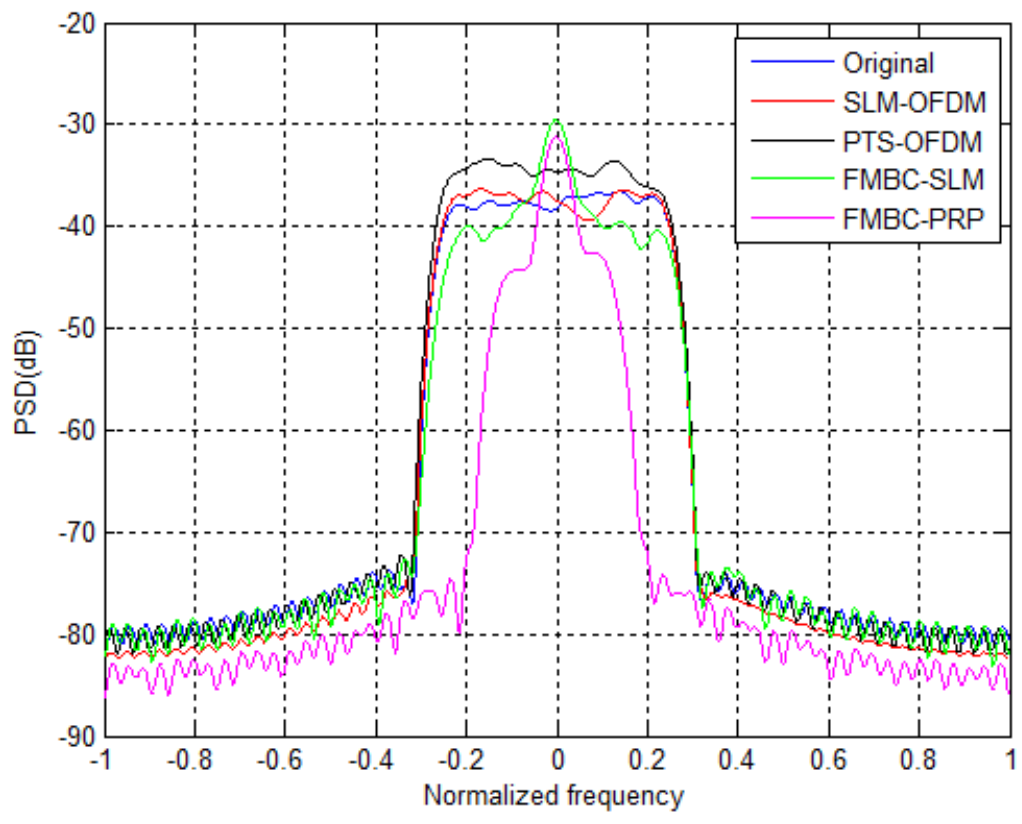

Figure5. OBI performance with $N=256$ subcarriers

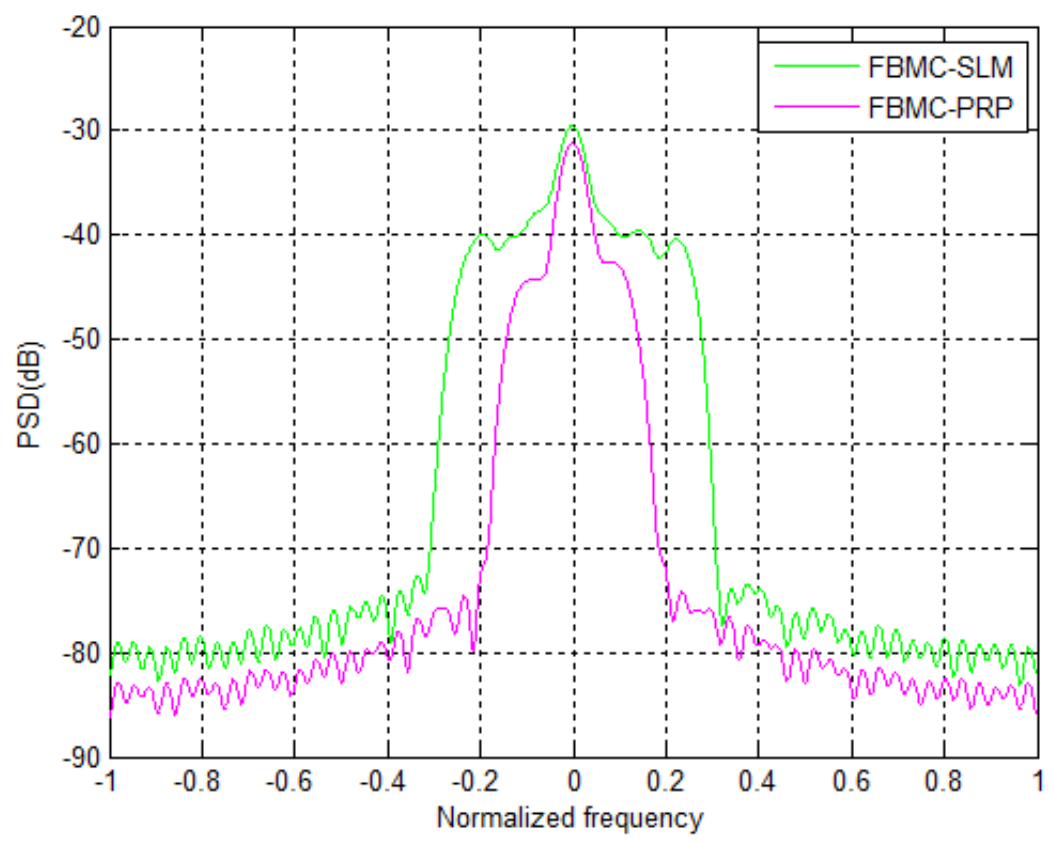

Figure6. OBI performance and comparison with FBMC-SLM

\section{Conclusion}

A PAPR reduction approach for FBMC OFDM/OQAM system is proposed in this paper, the method is simpler in implementation as it involves the summation of the previous blocks ith the current blocks of the symbol. Experimental show that the proposed approach could attain a considerable better performance when compared against the traditional OFDM approaches like SLM, PTS and FBMC based SLM approach. The method can be further improved by introducing the new phase sequences and testing under different channeling environments. 


\section{REFERENCES}

[1] B. Farhang-Boroujeny, "OFDM versus filter bank multicarrier," IEEE Signal Processing Magazine, vol. 28, no. 3, pp. 92-112, 2011

[2] D. Chen, D. Qu, and T. Jiang, "Prototype filter optimization to minimize stop band energy with NPR constraint for filter bank multicarrier modulation systems," IEEE Trans. Signal Process., vol. 61, no. 1, pp. 159-169, Jan. 2013.

[3] P. Siohan, C. Siclet, and N. Lacaille, "Analysis and design of OFDM/OQAM systems based on filterbank theory,” IEEE Trans. Signal Process., vol. 50, no. 5, pp. 1170-1183, May 2002.

[4] M. U. Rahim, T. H. Stitz, and M. Renfors, "Analysis of clipping-based PAPR-reduction in multicarrier systems," in Proc. IEEE VTC, Barcelona, Spain, Apr. 2009, pp. 1-5.

[5] Z. Kollar and P. Horvath, "PAPR reduction of FBMC by clipping and its iterative compensation," J. Comput. Netw. Communication, vol. 2012, pp. 1-11, Feb. 2012.

[6] A. Skrzypczak, J. Javaudin, and P. Sinhan, "Reduction of the peak-to average power ratio for the OFDM/OQAM modulation,” in Proc. IEEE VTC, Melbourne, Vic., Australia, May 2006, pp. 2018-2022

[7] C. H. Yuen, P. Amini, and B. Farhang-Boroujeny, "Single carrier frequency division multiple access (SC-FDMA) for filter bank multicarrier communication systems," in Proc. IEEE CROWNCOM, Cannes, France, Jun. 2010

[8] S. Lu, D. Qu, and Y. He, 'Sliding window tone reservation technique for the peak-to-average power ratio reduction of FBMC-OQAM signals," IEEE Wireless Commun. Lett., vol. 1, no. 4, pp. 268271, Aug. 2012.

[9] V. S. Kumar and S. Anuradha, "Sliding window tone reservation using smart gradient projection method for PAPR reduction of FBMC signals," in Proc. Int. Conf. Elect., Electron., Signals, Commun. Optim., Jan. 2015, pp. 494499.

[10] R. B"auml, R. F. H. Fischer and J. B. Huber, "Reducing the Peak-to- Average Power Ratio of Multicarrier Modulation by Selected Mapping," IEE Electronics Letters, Vol. 32, No. 22, pp. 2056-2057, Oct. 1996.

[11] S. H. M"uller and J. B. Huber, "OFDM with Reduced Peak-to-Average Power Ratio by Optimum Combination of Partial Transmit Sequences," IEE Electronics Letters, Vol. 33, No. 5, pp. 368-369, Feb. 1997.

[12] T. Jiang, C. Ni, C. Ye, Y. Wu, and K. Luo, "A novel multi-block tone reservation scheme for PAPR reduction in OQAM-OFDM systems," IEEE Trans. Broadcast., vol. 61, no. 4, pp. 717723, Dec. 2015. 